\title{
Nonexistence of Homoclinic Orbits for a Class of Hamiltonian Systems
}

\author{
Xiaoyan Lin, ${ }^{1}$ Qi-Ming Zhang, ${ }^{2}$ and X. H. Tang ${ }^{3}$ \\ ${ }^{1}$ Department of Mathematics, Huaihua College, Huaihua, Hunan 418008, China \\ ${ }^{2}$ College of Science, Hunan University of Technology, Zhuzhou, Hunan 412007, China \\ ${ }^{3}$ School of Mathematical Sciences and Computing Technology, Central South University, Changsha, Hunan 410083, China
}

Correspondence should be addressed to Xiaoyan Lin; xiaoyanlin98@hotmail.com

Received 31 March 2013; Accepted 22 July 2013

Academic Editor: Marco Squassina

Copyright (C) 2013 Xiaoyan Lin et al. This is an open access article distributed under the Creative Commons Attribution License, which permits unrestricted use, distribution, and reproduction in any medium, provided the original work is properly cited.

We give several sufficient conditions under which the first-order nonlinear Hamiltonian system $x^{\prime}(t)=\alpha(t) x(t)+f(t, y(t)), y^{\prime}(t)=$ $-g(t, x(t))-\alpha(t) y(t)$ has no solution $(x(t), y(t))$ satisfying condition $0<\int_{-\infty}^{+\infty}\left[|x(t)|^{\nu}+(1+\beta(t))|y(t)|^{\mu}\right] d t<+\infty$, where $\mu, v>1$ and $(1 / \mu)+(1 / \nu)=1,0 \leq x f(t, x) \leq \beta(t)|x|^{\mu}, x g(t, x) \leq \gamma_{0}(t)|x|^{\nu}, \beta(t), \gamma_{0}(t) \geq 0$, and $\alpha(t)$ are locally Lebesgue integrable real-valued functions defined on $\mathbb{R}$.

\section{Introduction}

In 1897, Poincaré [1] studied the existence of homoclinic solutions for Hamiltonian systems and realized that homoclinic solutions play a very important role in the study of the behavior of dynamical systems. Since then many methods have been developed to this study ([2-6]). Recently, the critical point theory has been successfully applied to establish the existence and multiplicity of homoclinic solutions for Hamiltonian systems; see [1,7-20] and references therein.

Among the above-mentioned literature, there are two classes of Hamiltonian systems that have been widely investigated: one is the second-order Hamiltonian system

$$
\left(p(t) u^{\prime}(t)\right)^{\prime}-L(t) u(t)+\nabla W(t, u(t))=0
$$

and the other is the first-order Hamiltonian system

$$
\left(\begin{array}{l}
x^{\prime}(t) \\
y^{\prime}(t)
\end{array}\right)=\left(\begin{array}{cc}
0 & -I \\
I & 0
\end{array}\right)\left(\begin{array}{l}
H_{x}(t, x(t), y(t)) \\
H_{y}(t, x(t), y(t))
\end{array}\right) .
$$

By means of variational methods, in order to seek the homoclinic solutions for system (1), one usually defines a functional $\varphi(u)$ on the Banach space

$$
\begin{aligned}
& E=\left\{u \in W^{1,2}\left(\mathbb{R}, \mathbb{R}^{n}\right):\right. \\
&\left.\int_{\mathbb{R}}\left[a_{1}(t)\left|u^{\prime}(t)\right|^{2}+a_{2}(t)|u(t)|^{2}\right] d t<+\infty\right\},
\end{aligned}
$$

where $a_{1}, a_{2} \in C(\mathbb{R},(0,+\infty))$ associated with the coefficients $p(t)$ and $L(t)$ of system (1). And then one proves that $\varphi$ possesses critical points on $E$ which are homoclinic solutions of system (1). Thus, the nontrivial homoclinic solutions of system (1) which were studied in the existing work are actually a class of special solutions satisfying condition

$$
0<\int_{\mathbb{R}}\left[\left(1+a_{1}(t)\right)\left|u^{\prime}(t)\right|^{2}+\left(1+a_{2}(t)\right)|u(t)|^{2}\right] d t<+\infty
$$

Similarly, the non-trivial homoclinic solutions of system (2) which were studied in the literature are also a class of special solutions satisfying condition

$$
0<\int_{\mathbb{R}}\left[\left(1+b_{1}(t)\right)|x(t)|^{2}+\left(1+b_{2}(t)\right)|y(t)|^{2}\right] d t<+\infty,
$$


where $b_{1}, b_{2} \in C(\mathbb{R},(0,+\infty))$ associated with the potential $H$ of system (2).

As mentioned earlier, the existence and multiplicity of homoclinic solutions for Hamiltonian systems have been studied extensively via critical point theory in recent years; various sufficient conditions for existence are established. However, as we know, there are no results on nonexistence of homoclinic solutions for Hamiltonian systems in the literature. For the simplest second-order Hamiltonian system,

$$
x^{\prime \prime}(t)+q(t) x(t)=0
$$

has no non-trivial homoclinic solutions as $q(t) \equiv$ constant, but when $q(t) \quad \equiv$ constant, there seem to be no results on existence or non-existence of homoclinic solutions in the literature either.

In this paper, we consider the first-order nonlinear Hamiltonian system

$$
\begin{gathered}
x^{\prime}(t)=\alpha(t) x(t)+f(t, y(t)), \\
y^{\prime}(t)=-g(t, x(t))-\alpha(t) y(t),
\end{gathered}
$$

where $\alpha(t)$ is locally Lebesgue integrable real-valued function defined on $\mathbb{R}, f, g: \mathbb{R}^{2} \rightarrow \mathbb{R}$. For every $t \in \mathbb{R}, f(t, x)$ and $g(t, x)$ are continuous on $x$ in $\mathbb{R}$, and for every $x \in \mathbb{R}$, $f(t, x)$ and $g(t, x)$ are locally Lebesgue integrable real-valued functions on $t$.

For the sake of convenience, we give the following assumptions on $f$ and $g$.

(F) For any $c \neq 0$, meas $\left\{t \in \mathbb{R}: f\left(t, c \exp \left(-\int_{0}^{t} \alpha(s) d s\right)\right)\right.$ $\neq 0\}>0$, and there exist a constant $\mu>1$ and a locally Lebesgue integrable nonnegative function $\beta(t)$ defined on $\mathbb{R}$ such that

$$
0 \leq x f(t, x) \leq \beta(t)|x|^{\mu}, \quad \forall(t, x) \in \mathbb{R}^{2} .
$$

(G) $g(t, 0)=0$ for $t \in \mathbb{R}$, and there exists a locally Lebesgue integrable nonnegative function $\gamma_{0}(t)$ defined on $\mathbb{R}$ such that

$$
x g(t, x) \leq \gamma_{0}(t)|x|^{\nu}, \quad \forall(t, x) \in \mathbb{R}^{2},
$$

where $\nu>1$ and $(1 / \mu)+(1 / \nu)=1$.

Remark 1. In case $g(t, x)=\gamma(t) \varphi(x)$, where $\gamma(t)$ is locally Lebesgue integrable real-valued function defined on $\mathbb{R}, \varphi \in$ $C(\mathbb{R}, \mathbb{R})$, and satisfies that

$$
0 \leq x \varphi(x) \leq|x|^{\nu}, \quad \forall(t, x) \in \mathbb{R}^{2},
$$

then we can choose $\gamma_{0}(t)=\gamma^{+}(t)=\max \{\gamma(t), 0\}$.

$$
\begin{aligned}
& \text { Let } u(t)=(x(t), y(t))^{\top}, H(t, x, y)=\int_{0}^{x} g(t, s) d s+ \\
& \alpha(t) x y+\int_{0}^{y} f(t, s) d s \text {, and } \\
& J=\left(\begin{array}{cc}
0 & 1 \\
-1 & 0
\end{array}\right) .
\end{aligned}
$$

Then we can rewrite (7) as a standard first-order Hamiltonian system

$$
u^{\prime}(t)=J \nabla H(t, u(t))
$$

There are two special forms of system (7) which have been dealt with extensively in the literature: one is the first-order linear Hamiltonian system

$$
\begin{aligned}
& x^{\prime}(t)=\alpha(t) x(t)+\beta(t) y(t), \\
& y^{\prime}(t)=-\gamma(t) x(t)-\alpha(t) y(t)
\end{aligned}
$$

and the other is the first-order quasilinear Hamiltonian system

$$
\begin{gathered}
x^{\prime}(t)=\alpha(t) x(t)+\beta(t)|y(t)|^{\mu-2} y(t), \\
y^{\prime}(t)=-\gamma(t)|x(t)|^{\nu-2} x(t)-\alpha(t) y(t),
\end{gathered}
$$

(see $[21,22]$ and the references therein), where $\mu, \nu>1$ and $(1 / \mu)+(1 / \nu)=1$, and $\beta(t)$ and $\gamma(t)$ are locally Lebesgue integrable real-valued functions defined on $\mathbb{R}$. In addition, the special forms of system (7) also contain many other well-known second-order differential equations such as the second-order linear differential equation

$$
\left(p(t) x^{\prime}(t)\right)^{\prime}+q(t) x(t)=0,
$$

the second-order half-linear differential equation

$$
\left[p(t)\left|x^{\prime}(t)\right|^{r-2} x^{\prime}(t)\right]^{\prime}+q(t)|x(t)|^{r-2} x(t)=0,
$$

and the second-order nonlinear differential equation

$$
\left[p(t) \phi\left(x^{\prime}(t)\right)\right]^{\prime}+h(t, x(t))=0,
$$

where $r>1, p(t)$ and $q(t)$ are locally Lebesgue integrable realvalued functions defined on $\mathbb{R}$ and $p(t)>0, \phi \in C(\mathbb{R}, \mathbb{R})$, and $h \in C\left(\mathbb{R}^{2}, \mathbb{R}\right)$. Indeed, we can rewrite the above-mentioned second-order differential equations as the form of system (7). For example, let

$$
y(t)=p(t)\left|x^{\prime}(t)\right|^{r-2} x^{\prime}(t) .
$$

Then (16) can be written as the form of (13):

$$
\begin{gathered}
x^{\prime}(t)=[p(t)]^{1 /(1-r)}|y(t)|^{(2-r) /(r-1)} y(t), \\
y^{\prime}(t)=-q(t)|x(t)|^{r-2} x(t),
\end{gathered}
$$

where $\mu=r /(r-1), \nu=r$ and $\alpha(t)=0, \beta(t)=[p(t)]^{1 /(1-r)}$, and $\gamma(t)=q(t)$. If $\phi$ has an inverse $\phi^{-1}$, then let

$$
y(t)=p(t) \phi\left(x^{\prime}(t)\right) .
$$


Hence, (17) can be written as the form of (7):

$$
\begin{aligned}
& x^{\prime}(t)=\phi^{-1}\left(\frac{y(t)}{p(t)}\right), \\
& y^{\prime}(t)=-h(t, x(t)),
\end{aligned}
$$

where $\alpha(t)=0, f(t, x)=\phi^{-1}(x / p(t))$, and $g(t, x)=h(t, x)$.

In Sections 2 and 3, we will give some necessary conditions for existence of homoclinic solutions of systems (7) and (13), which satisfy conditions

$$
\begin{aligned}
& 0<\int_{-\infty}^{+\infty}\left[|x(t)|^{\nu}+(1+\beta(t))|y(t)|^{\mu}\right] d t<+\infty, \\
& 0<\int_{-\infty}^{+\infty}\left[|x(t)|^{2}+(1+\beta(t))|y(t)|^{2}\right] d t<+\infty,
\end{aligned}
$$

respectively. These necessary conditions are actually Lyapunov-type inequalities, which generalize the classical Lyapunov inequality for system (6); see [21-25]. Taking advantage of these necessary conditions, we are able to establish some criteria for non-existence of homoclinic solutions of systems (7) and (13) in Section 4.

\section{Lyapunov-Type Inequalities for System (7)}

In this section, we will establish some Lyapunov-type inequalities for system (7). For the sake of convenience, we list some assumptions on $\alpha(t)$ and $\beta(t)$ as follows:

(A0) $\liminf \operatorname{int}_{|t| \rightarrow+\infty} \int_{0}^{t} \alpha(s) d s>-\infty$,

(A1) $\int_{-\infty}^{+\infty}|\alpha(s)| d s<+\infty$,

(B0) $\beta(t) \geq(\not \equiv) 0, \forall t \in \mathbb{R}$,

(B1) $\beta(t)>0, \forall t \in \mathbb{R}$,

(B2) $\int_{-\infty}^{+\infty} \beta(\tau) \exp \left(-\mu \int_{0}^{\tau} \alpha(s) d s\right) d \tau<+\infty$.

Denote

$$
\begin{gathered}
\zeta(t):=\left[\int_{-\infty}^{t} \beta(\tau) \exp \left(\mu \int_{\tau}^{t} \alpha(s) d s\right) d \tau\right]^{\nu / \mu}, \\
\eta(t):=\left[\int_{t}^{+\infty} \beta(\tau) \exp \left(-\mu \int_{t}^{\tau} \alpha(s) d s\right) d \tau\right]^{\nu / \mu} .
\end{gathered}
$$

Theorem 2. Suppose that hypotheses (F), (G), (AO), (B0), and (B2) are satisfied. If system (7) has a solution $(x(t), y(t))$ satisfying

$$
0<\int_{-\infty}^{+\infty}\left[|x(t)|^{\nu}+(1+\beta(t))|y(t)|^{\mu}\right] d t<+\infty
$$

then one has the following inequality:

$$
\int_{-\infty}^{+\infty} \frac{\zeta(t) \eta(t)}{\zeta(t)+\eta(t)} \gamma_{0}(t) d t \geq 1
$$

Proof. Hypothesis (B2) implies that functions $\zeta(t)$ and $\eta(t)$ are well defined on $\mathbb{R}$. Without loss of generality, we can assume that

$$
\int_{-\infty}^{+\infty} \frac{\zeta(t) \eta(t)}{\zeta(t)+\eta(t)} \gamma_{0}(t) d t<+\infty
$$

It follows from (F), (25), and (B0) that

$$
\begin{gathered}
\liminf _{t \rightarrow-\infty}|x(t)|=\liminf _{t \rightarrow+\infty}|x(t)|=0, \\
\liminf _{t \rightarrow-\infty}|y(t)|=\liminf _{t \rightarrow+\infty}|y(t)|=0, \\
\int_{-\infty}^{+\infty} f(\tau, y(\tau)) y(\tau) d \tau \leq \int_{-\infty}^{+\infty} \beta(\tau)|y(\tau)|^{\mu} d \tau<+\infty .
\end{gathered}
$$

Set $A(t)=\{\tau \in(-\infty, t]: \beta(\tau)>0\}$ for $t \in \mathbb{R}$, and then it follows from $(F)$ that

$$
\begin{aligned}
& {[\beta(\tau)]^{-v / \mu}|f(\tau, z)|^{\nu}} \\
& \quad=|z|^{-\nu}[\beta(\tau)]^{-v / \mu}|z f(\tau, z)|^{\nu} \\
& \quad \leq\left.\left.|z|^{-\nu}[\beta(\tau)]^{-v / \mu}|\beta(\tau)| z\right|^{\mu}\right|^{\nu-1} z f(\tau, z) \\
& \quad=z f(\tau, z), \quad \tau \in A(t), t \in \mathbb{R}, \quad z \neq 0 .
\end{aligned}
$$

Since $f(\tau, 0)=0$ for $\tau \in \mathbb{R}$, it follows that

$$
[\beta(\tau)]^{-\nu / \mu}|f(\tau, z)|^{\nu} \leq z f(\tau, z), \quad \tau \in A(t), t \in \mathbb{R} .
$$

Hence, from (F), (23), (24), (30), (32), and the Hölder inequality, one has

$$
\begin{aligned}
\int_{-\infty}^{t}|f(\tau, y(\tau))| \exp \left(\int_{\tau}^{t} \alpha(s) d s\right) d \tau \\
=\int_{A(t)}|f(\tau, y(\tau))| \exp \left(\int_{\tau}^{t} \alpha(s) d s\right) d \tau \\
\leq\left[\int_{A(t)} \beta(\tau) \exp \left(\mu \int_{\tau}^{t} \alpha(s) d s\right) d \tau\right]^{1 / \mu} \\
\quad \times\left[\int_{A(t)}[\beta(\tau)]^{-v / \mu}|f(\tau, y(\tau))|^{\nu} d \tau\right]^{1 / \nu} \\
\leq\left[\int_{A(t)} \beta(\tau) \exp \left(\mu \int_{\tau}^{t} \alpha(s) d s\right) d \tau\right]^{1 / \mu}
\end{aligned}
$$




$$
\begin{aligned}
& \times\left[\int_{A(t)} f(\tau, y(\tau)) y(\tau) d \tau\right]^{1 / v} \\
& \leq {\left[\int_{-\infty}^{t} \beta(\tau) \exp \left(\mu \int_{\tau}^{t} \alpha(s) d s\right) d \tau\right]^{1 / \mu} } \\
& \times\left[\int_{-\infty}^{t} f(\tau, y(\tau)) y(\tau) d \tau\right]^{1 / v} \\
&= {[\zeta(t)]^{1 / v}\left[\int_{-\infty}^{t} f(\tau, y(\tau)) y(\tau) d \tau\right]^{1 / v} } \\
&<+\infty, \quad \forall t \in \mathbb{R}, \\
& \int_{t}^{+\infty}|f(\tau, y(\tau))| \exp \left(-\int_{t}^{\tau} \alpha(s) d s\right) d \tau \\
& \leq\left[\int_{t}^{+\infty} \beta(\tau) \exp \left(-\mu \int_{t}^{\tau} \alpha(s) d s\right) d \tau\right]^{1 / \mu} \\
& \times\left[\int_{t}^{+\infty} f(\tau, y(\tau)) y(\tau) d \tau\right]^{1 / v} \\
&=[\eta(t)]^{1 / v}\left[\int_{t}^{+\infty} f(\tau, y(\tau)) y(\tau) d \tau\right]^{1 / v} \\
&<+\infty, \quad \forall t \in \mathbb{R} .
\end{aligned}
$$

From (A0), (28), (33), (34), and the first equation of system (7), we have

$$
\begin{aligned}
& x(-\infty):=\lim _{t \rightarrow-\infty} x(t)=0=\lim _{t \rightarrow+\infty} x(t):=x(+\infty), \\
& x(t)=\int_{-\infty}^{t} f(\tau, y(\tau)) \exp \left(\int_{\tau}^{t} \alpha(s) d s\right) d \tau, \quad \forall t \in \mathbb{R},
\end{aligned}
$$

$$
x(t)=-\int_{t}^{+\infty} f(\tau, y(\tau)) \exp \left(-\int_{t}^{\tau} \alpha(s) d s\right) d \tau, \quad \forall t \in \mathbb{R} .
$$

Combining (33) with (36), one has

$$
\begin{aligned}
|x(t)|^{\nu} & =\left|\int_{-\infty}^{t} f(\tau, y(\tau)) \exp \left(\int_{\tau}^{t} \alpha(s) d s\right) d \tau\right|^{\nu} \\
& \leq \zeta(t) \int_{-\infty}^{t} f(\tau, y(\tau)) y(\tau) d \tau, \quad \forall t \in \mathbb{R} .
\end{aligned}
$$

Similarly, it follows from (34) and (37) that

$$
\begin{aligned}
|x(t)|^{\nu} & =\left|\int_{t}^{+\infty} f(\tau, y(\tau)) \exp \left(-\int_{\tau}^{t} \alpha(s) d s\right) d \tau\right|^{\nu} \\
& \leq \eta(t) \int_{t}^{+\infty} f(\tau, y(\tau)) y(\tau) d \tau, \quad \forall t \in \mathbb{R} .
\end{aligned}
$$

Hence, from (38) and (39), one has

$$
|x(t)|^{\nu} \leq \frac{\zeta(t) \eta(t)}{\zeta(t)+\eta(t)} \int_{-\infty}^{+\infty} f(\tau, y(\tau)) y(\tau) d \tau, \quad \forall t \in \mathbb{R} .
$$

Now, it follows from (27), (30), and (40) that

$$
\begin{aligned}
\int_{-\infty}^{+\infty} & \gamma_{0}(t)|x(t)|^{\nu} d t \\
& \leq \int_{-\infty}^{+\infty} \frac{\zeta(t) \eta(t)}{\zeta(t)+\eta(t)} \gamma_{0}(t) d t \int_{-\infty}^{+\infty} f(t, y(t)) y(t) d t \\
& <+\infty .
\end{aligned}
$$

By (29), we can choose two sequences $\left\{t_{-n}\right\}_{n=1}^{\infty}$ and $\left\{t_{n}\right\}_{n=1}^{\infty}$ such that

$$
-\infty<\cdots<t_{-3}<t_{-2}<t_{-1}<t_{1}<t_{2}<t_{3}<\cdots<+\infty,
$$

$$
\begin{gathered}
\lim _{n \rightarrow \infty} t_{-n}=-\infty, \quad \lim _{n \rightarrow \infty} t_{n}=+\infty, \\
\lim _{n \rightarrow \infty} y\left(t_{-n}\right)=\lim _{n \rightarrow \infty} y\left(t_{n}\right)=0 .
\end{gathered}
$$

By (7), we obtain

$$
(x(t) y(t))^{\prime}=f(t, y(t)) y(t)-g(t, x(t)) x(t) .
$$

Integrating the above equation from $t_{-n}$ to $t_{n}$, we have

$$
\begin{aligned}
& \int_{t_{-n}}^{t_{n}} g(t, x(t)) x(t) d t \\
&=\int_{t_{-n}}^{t_{n}} f(t, y(t)) y(t) d t+x\left(t_{-n}\right) y\left(t_{-n}\right) \\
& \quad-x\left(t_{n}\right) y\left(t_{n}\right), \quad n=1,2,3, \ldots
\end{aligned}
$$

Let $n \rightarrow \infty$ in the above equation, and using (30), (35), and (43) we obtain

$$
\lim _{n \rightarrow \infty} \int_{t_{-n}}^{t_{n}} g(t, x(t)) x(t) d t=\int_{-\infty}^{+\infty} f(t, y(t)) y(t) d t
$$

which, together with (41), implies that

$$
\begin{aligned}
& \int_{-\infty}^{+\infty} \gamma_{0}(t)|x(t)|^{\nu} d t \\
& \leq \int_{-\infty}^{+\infty} \frac{\zeta(t) \eta(t)}{\zeta(t)+\eta(t)} \gamma_{0}(t) d t \int_{-\infty}^{+\infty} f(t, y(t)) y(t) d t \\
&= {\left[\int_{-\infty}^{+\infty} \frac{\zeta(t) \eta(t)}{\zeta(t)+\eta(t)} \gamma_{0}(t) d t\right] } \\
& \times \lim _{n \rightarrow \infty} \int_{t_{-n}}^{t_{n}} g(t, x(t)) x(t) d t \\
& \leq {\left[\int_{-\infty}^{+\infty} \frac{\zeta(t) \eta(t)}{\zeta(t)+\eta(t)} \gamma_{0}(t) d t\right] } \\
& \times \lim _{n \rightarrow \infty} \int_{t_{-n}}^{t_{n}} \gamma_{0}(t)|x(t)|^{\nu} d t \\
&= \int_{-\infty}^{+\infty} \frac{\zeta(t) \eta(t)}{\zeta(t)+\eta(t)} \gamma_{0}(t) d t \int_{-\infty}^{+\infty} \gamma_{0}(t)|x(t)|^{\nu} d t .
\end{aligned}
$$


We claim that

$$
\int_{-\infty}^{+\infty} \gamma_{0}(t)|x(t)|^{v} d t>0
$$

If (48) is not true, then

$$
\int_{-\infty}^{+\infty} \gamma_{0}(t)|x(t)|^{v} d t=0
$$

From (F), (G), (46), and (49), we have

$$
\begin{aligned}
0 & \leq \int_{-\infty}^{+\infty} f(t, y(t)) y(t) d t \\
& =\lim _{n \rightarrow \infty} \int_{t_{-n}}^{t_{n}} g(t, x(t)) x(t) d t \\
& \leq \int_{-\infty}^{+\infty} \gamma_{0}(t)|x(t)|^{\nu} d t=0,
\end{aligned}
$$

which, together with (F), implies that

$$
f(t, y(t)) y(t)=0, \quad \text { a.e. } t \in \mathbb{R} \text {. }
$$

Combining (36) with (51), we obtain that

$$
x(t) \equiv 0, \quad \forall t \in \mathbb{R},
$$

which, together with $(\mathrm{G})$ and the second equation of system (7), implies that

$$
y(t)=y(0) \exp \left(-\int_{0}^{t} \alpha(s) d s\right), \quad \forall t \in \mathbb{R} .
$$

From (F), (51), and the above, one has

$$
y(t) \equiv 0, \quad \forall t \in \mathbb{R} .
$$

Both (52) and (54) contradict (25). Therefore, (48) holds. Hence, it follows from (47) and (48) that (26) holds.
Corollary 3. Suppose that hypotheses (F), (G), (A1), (B0), and (B2) are satisfied. If system (7) has a solution $(x(t), y(t))$ satisfying (25), then one has the following inequalities:

$$
\begin{aligned}
& \int_{-\infty}^{+\infty} \tilde{\gamma}(t)\left(\int_{-\infty}^{t} \tilde{\beta}(\tau) d \tau \int_{t}^{+\infty} \tilde{\beta}(\tau) d \tau\right)^{\nu / 2 \mu} d t \\
& \geq 2 \exp \left(-\frac{\nu}{2} \int_{-\infty}^{+\infty}\left(|\alpha(s)|+\mu^{-1}|\omega(s)|\right) d s\right) \\
& \left(\int_{-\infty}^{+\infty} \tilde{\beta}(t) d t\right)^{1 / \mu}\left(\int_{-\infty}^{+\infty} \tilde{\gamma}^{+}(t) d t\right)^{1 / \nu} \\
& \geq 2 \exp \left(-\frac{1}{2} \int_{-\infty}^{+\infty}\left(|\alpha(s)|+\mu^{-1}|\omega(s)|\right) d s\right) \\
& \int_{-\infty}^{+\infty} \gamma_{0}(t)\left(\int_{-\infty}^{t} \beta(\tau) d \tau \int_{t}^{+\infty} \beta(\tau) d \tau\right)^{v / 2 \mu} d t \\
& \geq 2 \exp \left(-\frac{\nu}{2} \int_{-\infty}^{+\infty}|\alpha(s)| d s\right) \\
& \left(\int_{-\infty}^{+\infty} \beta(t) d t\right)^{1 / \mu}\left(\int_{-\infty}^{+\infty} \gamma_{0}(t) d t\right)^{1 / \nu} \\
& \geq 2 \exp \left(-\frac{1}{2} \int_{-\infty}^{+\infty}|\alpha(s)| d s\right)
\end{aligned}
$$

where $\omega \in L^{1}(\mathbb{R})$ is an arbitrary function and

$$
\begin{aligned}
& \tilde{\beta}(t)=\beta(t) \exp \left(\int_{t_{0}}^{t} \omega(s) d s\right), \\
& \tilde{\gamma}(t)=\gamma_{0}(t) \exp \left(-\frac{\nu}{\mu} \int_{t_{0}}^{t} \omega(s) d s\right)
\end{aligned}
$$

for some $t_{0} \in \mathbb{R}$.

Proof. (A1), (B0), and (B2) imply that (A0) and $\int_{-\infty}^{+\infty} \beta(s) d s<$ $+\infty$. Since

$$
\zeta(t)+\eta(t) \geq 2[\zeta(t) \eta(t)]^{1 / 2}
$$

then it follows from (23), (24), (26), (56), and (57) that

$$
\begin{aligned}
1 & \leq \int_{-\infty}^{+\infty} \frac{\zeta(t) \eta(t)}{\zeta(t)+\eta(t)} \gamma_{0}(t) d t \\
& \leq \frac{1}{2} \int_{-\infty}^{+\infty}[\zeta(t) \eta(t)]^{1 / 2} \gamma_{0}(t) d t
\end{aligned}
$$




$$
\begin{aligned}
& =\frac{1}{2} \int_{-\infty}^{+\infty} \gamma_{0}(t)\left[\int_{-\infty}^{t} \beta(\tau) \exp \left(\mu \int_{\tau}^{t} \alpha(s) d s\right) d \tau\right. \\
& \times \int_{t}^{+\infty} \beta(\tau) \\
& \left.\times \exp \left(-\mu \int_{t}^{\tau} \alpha(s) d s\right) d \tau\right]^{\nu / 2 \mu} d t \\
& \leq \frac{1}{2}\left[\int_{-\infty}^{+\infty} \gamma_{0}(t)\left(\int_{-\infty}^{t} \beta(\tau) d \tau \int_{t}^{+\infty} \beta(\tau) d \tau\right)^{\nu / 2 \mu} d t\right] \\
& \quad \times \exp \left(\frac{\nu}{2} \int_{-\infty}^{+\infty}|\alpha(s)| d s\right) \\
& \leq \frac{1}{2}\left[\int_{-\infty}^{+\infty} \tilde{\gamma}(t)\left(\int_{-\infty}^{t} \tilde{\beta}(\tau) d \tau \int_{t}^{+\infty} \tilde{\beta}(\tau) d \tau\right)^{\nu / 2 \mu} d t\right] \\
& \quad \times \exp \left(\frac{\nu}{2} \int_{-\infty}^{+\infty}\left(|\alpha(s)|+\mu{ }^{-1}|\omega(s)|\right) d s\right),
\end{aligned}
$$

which implies that (55) holds. Note that

$$
\int_{-\infty}^{t} \tilde{\beta}(\tau) d \tau \int_{t}^{+\infty} \tilde{\beta}(\tau) d \tau \leq \frac{1}{4}\left(\int_{-\infty}^{+\infty} \tilde{\beta}(\tau) d \tau\right)^{2},
$$

which, together with (55), yields that (56) holds. It follows from (55) and (56) that (57) and (58) hold.

In case hypothesis (B0) is replaced by (B1) in the proof of Theorem 2, then (40) is strict; that is,

$$
|x(t)|^{\nu}<\frac{\zeta(t) \eta(t)}{\zeta(t)+\eta(t)} \int_{-\infty}^{+\infty} f(\tau, y(\tau)) y(\tau) d \tau, \quad \forall t \in \mathbb{R}
$$

In fact, if (63) is not true, then there exists a $t_{*} \in \mathbb{R}$ such that

$$
\left|x\left(t_{*}\right)\right|^{\nu}=\frac{\zeta\left(t_{*}\right) \eta\left(t_{*}\right)}{\zeta\left(t_{*}\right)+\eta\left(t_{*}\right)} \int_{-\infty}^{+\infty} f(\tau, y(\tau)) y(\tau) d \tau .
$$

Hence, from (38), (39), and (64), we obtain

$$
\begin{aligned}
& \left|x\left(t_{*}\right)\right|^{\nu}=\zeta\left(t_{*}\right) \int_{-\infty}^{t_{*}} f(\tau, y(\tau)) y(\tau) d \tau, \\
& \left|x\left(t_{*}\right)\right|^{\nu}=\eta\left(t_{*}\right) \int_{t_{*}}^{+\infty} f(\tau, y(\tau)) y(\tau) d \tau .
\end{aligned}
$$

It follows from (23), (38), and (65) that

$$
\begin{aligned}
\mid \int_{-\infty}^{t_{*}} f & \left.(\tau, y(\tau)) \exp \left(\int_{\tau}^{t_{*}} \alpha(s) d s\right) d \tau\right|^{\nu} \\
= & {\left[\int_{-\infty}^{t_{*}} \beta(\tau) \exp \left(\mu \int_{\tau}^{t_{*}} \alpha(s) d s\right) d \tau\right]^{\nu / \mu} } \\
& \times \int_{-\infty}^{t_{*}} f(\tau, y(\tau)) y(\tau) d \tau
\end{aligned}
$$

which, together with the Hölder inequality, implies that there exists a constant $c_{1}$ such that

$$
\begin{array}{r}
f(\tau, y(\tau)) y(\tau)=c_{1} \beta(\tau) \exp \left(\mu \int_{\tau}^{t_{*}} \alpha(s) d s\right) \\
-\infty<\tau \leq t_{*} .
\end{array}
$$

Similarly, it follows from (24), (39), (66), and the Hölder inequality that there exists a constant $c_{2}$ such that

$$
\begin{array}{r}
f(\tau, y(\tau)) y(\tau)=c_{2} \beta(\tau) \exp \left(-\mu \int_{t_{*}}^{\tau} \alpha(s) d s\right) \\
t_{*} \leq \tau<+\infty .
\end{array}
$$

From (F), (68), and (69), one has that $c_{1}, c_{2} \geq 0$. If $c_{1}=c_{2}=$ 0 , then $f(\tau, y(\tau)) y(\tau)=0$ for $\tau \in \mathbb{R}$; it follows from (36) that $x(t)=0$ for $t \in \mathbb{R}$. Similar to the proof of (54), one has $y(t)=0$ for $t \in \mathbb{R}$, which contradicts (25). If $c_{1}+c_{2}>0$, then $f(\tau, y(\tau)) y(\tau)>0$ for $\tau \in\left(-\infty, t_{*}\right]$ or for $\tau \in\left[t_{*},+\infty\right)$; it follows from (A0) and (36) that $x(+\infty) \neq 0$, which contradicts (35). The above two cases show that (63) holds. Hence, in view of the proof of Theorem 2, we have the following theorem.

Theorem 4. Suppose that hypotheses (F), (G), (A0), (B1), and (B2) are satisfied. If system (7) has a solution $(x(t), y(t))$ satisfying (25), then one has the following inequality:

$$
\int_{-\infty}^{+\infty} \frac{\zeta(t) \eta(t)}{\zeta(t)+\eta(t)} \gamma_{0}(t) d t>1
$$

where $\zeta(t)$ and $\eta(t)$ are defined by (23) and (24), respectively.

Similar to the proof of Corollary 3 , we can drive the following corollary from Theorem 4 .

Corollary 5. Suppose that hypotheses (F), (G), (A1), (B1), and (B2) are satisfied. If system (7) has a solution $(x(t), y(t))$ satisfying (25), then

$$
\begin{aligned}
\int_{-\infty}^{+\infty} \tilde{\gamma}(t)\left(\int_{-\infty}^{t} \tilde{\beta}(\tau) d \tau \int_{t}^{+\infty} \tilde{\beta}(\tau) d \tau\right)^{\nu / 2 \mu} d t \\
>2 \exp \left(-\frac{\nu}{2} \int_{-\infty}^{+\infty}\left(|\alpha(s)|+\mu^{-1}|\omega(s)|\right) d s\right) \\
\left(\int_{-\infty}^{+\infty} \tilde{\beta}(t) d t\right)^{1 / \mu}\left(\int_{-\infty}^{+\infty} \tilde{\gamma}(t) d t\right)^{1 / \nu} \\
>2 \exp \left(-\frac{1}{2} \int_{-\infty}^{+\infty}\left(|\alpha(s)|+\mu^{-1}|\omega(s)|\right) d s\right)
\end{aligned}
$$




$$
\begin{gathered}
\int_{-\infty}^{+\infty} \gamma_{0}(t)\left(\int_{-\infty}^{t} \beta(\tau) d \tau \int_{t}^{+\infty} \beta(\tau) d \tau\right)^{\nu / 2 \mu} d t \\
>2 \exp \left(-\frac{\nu}{2} \int_{-\infty}^{+\infty}|\alpha(s)| d s\right) \\
\left(\int_{-\infty}^{+\infty} \beta(t) d t\right)^{1 / \mu}\left(\int_{-\infty}^{+\infty} \gamma_{0}(t) d t\right)^{1 / \nu} \\
>2 \exp \left(-\frac{1}{2} \int_{-\infty}^{+\infty}|\alpha(s)| d s\right)
\end{gathered}
$$

If (17) has a solution $x(t)$ satisfying (73), then

$$
\begin{aligned}
& \int_{-\infty}^{+\infty} \frac{\left(\int_{-\infty}^{t}[p(\tau)]^{-1 /(r-1)} d \tau\right)^{r-1}\left(\int_{t}^{+\infty}[p(\tau)]^{-1 /(r-1)} d \tau\right)^{r-1}}{\left(\int_{-\infty}^{t}[p(\tau)]^{-1 /(r-1)} d \tau\right)^{r-1}+\left(\int_{t}^{+\infty}[p(\tau)]^{-1 /(r-1)} d \tau\right)^{r-1}} \\
& \quad \times \gamma_{0}(t) d t>1 .
\end{aligned}
$$

where $\widetilde{\beta}(t)$ and $\widetilde{\gamma}(t)$ are defined by (59).

Applying Theorem 4 and Corollary 5 to system (19) (i.e., (16)), we have immediately the following two corollaries.

Corollary 6. Suppose that $r>1, p(t)>0$ for $t \in \mathbb{R}$ and

$$
\int_{-\infty}^{+\infty} \frac{1}{[p(\tau)]^{1 /(r-1)}} d \tau<+\infty
$$

If (16) has a solution $x(t)$ satisfying

$$
\begin{aligned}
0 & <\int_{-\infty}^{+\infty}\left[|x(t)|^{r}+[p(t)]^{1 /(r-1)}(1+p(t))\left|x^{\prime}(t)\right|^{r}\right] d t \\
& <+\infty
\end{aligned}
$$

then

$$
\begin{aligned}
& \int_{-\infty}^{+\infty} \frac{\left(\int_{-\infty}^{t}[p(\tau)]^{-1 /(r-1)} d \tau\right)^{r-1}\left(\int_{t}^{+\infty}[p(\tau)]^{-1 /(r-1)} d \tau\right)^{r-1}}{\left(\int_{-\infty}^{t}[p(\tau)]^{-1 /(r-1)} d \tau\right)^{r-1}+\left(\int_{t}^{+\infty}[p(\tau)]^{-1 /(r-1)} d \tau\right)^{r-1}} \\
& \times q^{+}(t) d t>1 \\
& \int_{-\infty}^{+\infty} q^{+}(t)\left(\int_{-\infty}^{t}[p(\tau)]^{-1 /(r-1)} d \tau\right. \\
&\left.\times \int_{t}^{+\infty}[p(\tau)]^{-1 /(r-1)} d \tau\right)^{(r-1) / 2} d t>2 .
\end{aligned}
$$

Applying Theorem 4 to the second-order nonlinear differential equation (17) (i.e., system (21)), where $\alpha(t)=0$, $f(t, x)=\phi^{-1}(x / p(t))$, and $g(t, x)=h(t, x)$, we have the following corollary.

Corollary 7. Suppose that $r>1$ and $p(t)>0$ for $t \in \mathbb{R}$, and that (72) and the following hypothesis are satisfied:

(H1) There exists a locally Lebesgue integrable nonnegative function $\gamma_{0}(t)$ defined on $\mathbb{R}$ such that

$$
\begin{gathered}
0 \leq x \phi^{-1}(x) \leq|x|^{r /(r-1)}, \\
x h(t, x) \leq \gamma_{0}(t)|x|^{r}, \quad \forall(t, x) \in \mathbb{R}^{2} .
\end{gathered}
$$

\section{Lyapunov-Type Inequalities for System (13)}

When $\mu=\nu=2$, assumption (B2) reduces to the following form:

$$
\left(\mathrm{B} 2^{\prime}\right) \int_{-\infty}^{+\infty} \beta(\tau) \exp \left(-2 \int_{0}^{\tau} \alpha(s) d s\right) d \tau<+\infty
$$

Applying some results obtained in the last section to the first-order linear Hamiltonian system (13), we have immediately the following corollaries.

Corollary 8. Suppose that hypotheses (A0), (B0), and $\left(B 2^{\prime}\right)$ are satisfied. If system (13) has a solution $(x(t), y(t))$ satisfying

$$
0<\int_{-\infty}^{+\infty}\left[|x(t)|^{2}+(1+\beta(t))|y(t)|^{2}\right] d t<+\infty
$$

then

$$
\begin{aligned}
\int_{-\infty}^{+\infty}( & \left(\left[\int_{-\infty}^{t} \beta(\tau) \exp \left(2 \int_{\tau}^{t} \alpha(s) d s\right) d \tau\right]\right. \\
& \left.\times\left[\int_{t}^{+\infty} \beta(\tau) \exp \left(-2 \int_{t}^{\tau} \alpha(s) d s\right) d \tau\right]\right) \\
& \times\left(\int_{-\infty}^{t} \beta(\tau) \exp \left(2 \int_{\tau}^{t} \alpha(s) d s\right) d \tau\right. \\
& \left.\left.+\int_{t}^{+\infty} \beta(\tau) \exp \left(-2 \int_{t}^{\tau} \alpha(s) d s\right) d \tau\right)^{-1}\right) \\
& \times \gamma^{+}(t) d t \geq 1, \\
\int_{-\infty}^{+\infty} \gamma^{+}(t)\left(\int_{-\infty}^{t} \beta(\tau) d \tau \int_{t}^{+\infty} \beta(\tau) d \tau\right)^{1 / 2} d t & 2 \exp \left(-\int_{-\infty}^{+\infty}|\alpha(s)| d s\right) .
\end{aligned}
$$


Corollary 9. Suppose that hypotheses (A0), (B1), and $\left(B 2^{\prime}\right)$ are satisfied. If system (13) has a solution $(x(t), y(t))$ satisfying (77), then

$$
\begin{gathered}
\int_{-\infty}^{+\infty}\left(\left(\left[\int_{-\infty}^{t} \beta(\tau) \exp \left(2 \int_{\tau}^{t} \alpha(s) d s\right) d \tau\right]\right.\right. \\
\left.\times\left[\int_{t}^{+\infty} \beta(\tau) \exp \left(-2 \int_{t}^{\tau} \alpha(s) d s\right) d \tau\right]\right) \\
\times\left(\int_{-\infty}^{t} \beta(\tau) \exp \left(2 \int_{\tau}^{t} \alpha(s) d s\right) d \tau\right. \\
\left.\left.\quad+\int_{t}^{+\infty} \beta(\tau) \exp \left(-2 \int_{t}^{\tau} \alpha(s) d s\right) d \tau\right)^{-1}\right) \\
\times \gamma^{+}(t) d t>1, \\
\int_{-\infty}^{+\infty} \gamma^{+}(t)\left(\int_{-\infty}^{t} \beta(\tau) d \tau \int_{t}^{+\infty} \beta(\tau) d \tau\right)^{1 / 2} d t \\
>2 \exp \left(-\int_{-\infty}^{+\infty}|\alpha(s)| d s\right) .
\end{gathered}
$$

Corollary 10. Suppose that $p(t)>0$ for $t \in \mathbb{R}$ and that

$$
\int_{-\infty}^{+\infty} \frac{d \tau}{p(\tau)}<+\infty
$$

If (15) has a solution $x(t)$ satisfying

$$
0<\int_{-\infty}^{+\infty}\left[|x(t)|^{2}+p(t)(1+p(t))\left|x^{\prime}(t)\right|^{2}\right] d t<+\infty
$$

then

$$
\int_{-\infty}^{+\infty} q^{+}(t)\left(\int_{-\infty}^{t} \frac{d \tau}{p(\tau)} \int_{t}^{+\infty} \frac{d \tau}{p(\tau)}\right) d t>\int_{-\infty}^{+\infty} \frac{d \tau}{p(\tau)}
$$

Corollary 11. Suppose that $p(t)>0$ for $t \in \mathbb{R}$ and that (80) and the following hypothesis are satisfied:

(H2) There exists a locally Lebesgue integrable nonnegative function $\gamma_{0}(t)$ defined on $\mathbb{R}$ such that

$$
\begin{gathered}
0 \leq x \phi^{-1}(x) \leq|x|^{2}, \\
x h(t, x) \leq \gamma_{0}(t)|x|^{2}, \quad \forall(t, x) \in \mathbb{R}^{2} .
\end{gathered}
$$

If (17) has a solution $x(t)$ satisfying (81), then

$$
\int_{-\infty}^{+\infty} \gamma_{0}(t)\left(\int_{-\infty}^{t} \frac{d \tau}{p(\tau)} \int_{t}^{+\infty} \frac{d \tau}{p(\tau)}\right) d t>\int_{-\infty}^{+\infty} \frac{d \tau}{p(\tau)}
$$

\section{Nonexistence of Homoclinic Solutions}

Applying the results obtained in Sections 2 and 3, we can drive the following criteria for non-existence of homoclinic solutions of systems (7) and (13) immediately.
Corollary 12. Suppose that hypotheses (F), (G), (AO), (B0), and (B2) are satisfied. If one of the conditions

$$
\begin{gathered}
\int_{-\infty}^{+\infty} \frac{\zeta(t) \eta(t)}{\zeta(t)+\eta(t)} \gamma_{0}(t) d t<1, \\
\int_{-\infty}^{+\infty} \gamma_{0}(t)\left(\int_{-\infty}^{t} \beta(\tau) d \tau \int_{t}^{+\infty} \beta(\tau) d \tau\right)^{\nu / 2 \mu} d t \\
<2 \exp \left(-\frac{\nu}{2} \int_{-\infty}^{+\infty}|\alpha(s)| d s\right), \\
\left(\int_{-\infty}^{+\infty} \beta(t) d t\right)^{1 / \mu}\left(\int_{-\infty}^{+\infty} \gamma_{0}(t) d t\right)^{1 / \nu} \\
<2 \exp \left(-\frac{1}{2} \int_{-\infty}^{+\infty}|\alpha(s)| d s\right)
\end{gathered}
$$

holds, then system (7) has no solution $(x(t), y(t))$ satisfying

$$
0<\int_{-\infty}^{+\infty}\left[|x(t)|^{\nu}+(1+\beta(t))|y(t)|^{\mu}\right] d t<+\infty
$$

Corollary 13. Suppose that hypotheses (F), (G), (A0), (B1), and (B2) are satisfied. If one of the conditions

$$
\begin{gathered}
\int_{-\infty}^{+\infty} \frac{\zeta(t) \eta(t)}{\zeta(t)+\eta(t)} \gamma_{0}(t) d t \leq 1 \\
\int_{-\infty}^{+\infty} \gamma_{0}(t)\left(\int_{-\infty}^{t} \beta(\tau) d \tau \int_{t}^{+\infty} \beta(\tau) d \tau\right)^{\nu / 2 \mu} d t \\
\leq 2 \exp \left(-\frac{\nu}{2} \int_{-\infty}^{+\infty}|\alpha(s)| d s\right) \\
\left(\int_{-\infty}^{+\infty} \beta(t) d t\right)^{1 / \mu}\left(\int_{-\infty}^{+\infty} \gamma_{0}(t) d t\right)^{1 / \nu} \\
\leq 2 \exp \left(-\frac{1}{2} \int_{-\infty}^{+\infty}|\alpha(s)| d s\right)
\end{gathered}
$$

holds, then system (7) has no solution $(x(t), y(t))$ satisfying (86).

Corollary 14. Suppose that hypotheses (AO), (B0), and $\left(B 2^{\prime}\right)$ are satisfied. If

$$
\begin{aligned}
& \int_{-\infty}^{+\infty}\left(\left(\left[\int_{-\infty}^{t} \beta(\tau) \exp \left(2 \int_{\tau}^{t} \alpha(s) d s\right) d \tau\right]\right.\right. \\
& \left.\times\left[\int_{t}^{+\infty} \beta(\tau) \exp \left(-2 \int_{t}^{\tau} \alpha(s) d s\right) d \tau\right]\right) \\
& \times\left(\int_{-\infty}^{t} \beta(\tau) \exp \left(2 \int_{\tau}^{t} \alpha(s) d s\right) d \tau\right. \\
& \left.\left.\quad+\int_{t}^{+\infty} \beta(\tau) \exp \left(-2 \int_{t}^{\tau} \alpha(s) d s\right) d \tau\right)^{-1}\right) \\
& \times \gamma_{0}(t) d t<1
\end{aligned}
$$


or

$$
\begin{gathered}
\int_{-\infty}^{+\infty} \gamma^{+}(t)\left(\int_{-\infty}^{t} \beta(\tau) d \tau \int_{t}^{+\infty} \beta(\tau) d \tau\right)^{1 / 2} d t \\
\quad<2 \exp \left(-\int_{-\infty}^{+\infty}|\alpha(s)| d s\right)
\end{gathered}
$$

holds, then system (13) has no solution $(x(t), y(t))$ satisfying

$$
0<\int_{-\infty}^{+\infty}\left[|x(t)|^{2}+(1+\beta(t))|y(t)|^{2}\right] d t<+\infty
$$

Corollary 15. Suppose that hypotheses (A0), (B1), and $\left(B 2^{\prime}\right)$ are satisfied. If

$$
\begin{aligned}
\int_{-\infty}^{+\infty}(( & {\left[\int_{-\infty}^{t} \beta(\tau) \exp \left(2 \int_{\tau}^{t} \alpha(s) d s\right) d \tau\right] } \\
& \left.\times\left[\int_{t}^{+\infty} \beta(\tau) \exp \left(-2 \int_{t}^{\tau} \alpha(s) d s\right) d \tau\right]\right) \\
& \times\left(\int_{-\infty}^{t} \beta(\tau) \exp \left(2 \int_{\tau}^{t} \alpha(s) d s\right) d \tau\right. \\
& \left.\left.+\int_{t}^{+\infty} \beta(\tau) \exp \left(-2 \int_{t}^{\tau} \alpha(s) d s\right) d \tau\right)^{-1}\right)
\end{aligned}
$$$$
\times \gamma^{+}(t) d t \leq 1
$$

or

$$
\begin{aligned}
& \int_{-\infty}^{+\infty} \gamma^{+}(t)\left(\int_{-\infty}^{t} \beta(\tau) d \tau \int_{t}^{+\infty} \beta(\tau) d \tau\right)^{1 / 2} d t \\
& \quad \leq 2 \exp \left(-\int_{-\infty}^{+\infty}|\alpha(s)| d s\right)
\end{aligned}
$$

holds, then system (13) has no solution $(x(t), y(t))$ satisfying (92).

Corollary 16. Suppose that $p(t)>0$ for $t \in \mathbb{R}$ and that (80) holds. If

$$
\int_{-\infty}^{+\infty} q^{+}(t)\left(\int_{-\infty}^{t} \frac{d \tau}{p(\tau)} \int_{t}^{+\infty} \frac{d \tau}{p(\tau)}\right) d t \leq \int_{-\infty}^{+\infty} \frac{d \tau}{p(\tau)}
$$

then (15) has no solution $x(t)$ satisfying (81).

Corollary 17. Suppose that $p(t)>0$ for $t \in \mathbb{R}$ and that (80) and (H2) are satisfied. If

$$
\int_{-\infty}^{+\infty} \gamma_{0}(t)\left(\int_{-\infty}^{t} \frac{d \tau}{p(\tau)} \int_{t}^{+\infty} \frac{d \tau}{p(\tau)}\right) d t \leq \int_{-\infty}^{+\infty} \frac{d \tau}{p(\tau)}
$$

then (17) has no solution $x(t)$ satisfying (81).

Example 18. Consider the second-order nonlinear differential equation

$$
\left[\left(1+t^{2}\right) x^{\prime}(t)\right]^{\prime}+q(t) x(t)\left[1+\sin ^{2} x(t)\right]=0,
$$

where $q(t)$ is locally Lebesgue integrable real-valued function defined on $\mathbb{R}$. In view of Corollary 16 , if

$$
\int_{-\infty}^{+\infty}\left[\frac{\pi^{2}}{4}-(\arctan t)^{2}\right] q^{+}(t) d t \leq \frac{\pi}{2}
$$

then (97) has no solution $x(t)$ satisfying

$$
0<\int_{-\infty}^{+\infty}\left[|x(t)|^{2}+\left(1+t^{2}\right)^{2}\left|x^{\prime}(t)\right|^{2}\right] d t<+\infty .
$$

\section{Acknowledgments}

This work is partially supported by the NNSF (no. 11201138) of China Hunan Provincial Natural Science Foundation (no. 11JJ2005), and the Scientific Research Fund of Hunan Provincial Education Department (12B034).

\section{References}

[1] H. Poincaré, Les Méthodes Nouvelles de la Mécanique Céleste, Gauthier-Villars, Paris, France, 1897-1899.

[2] A. Ambrosetti and V. Coti Zelati, "Multiple homoclinic orbits for a class of conservative systems," Rendiconti del Seminario Matematico della Università di Padova, vol. 89, pp. 177-194, 1993.

[3] A. Ambrosetti and P. H. Rabinowitz, "Dual variational methods in critical point theory and applications," vol. 14, pp. 349-381, 1973.

[4] V. C. Zelati, I. Ekeland, and E. Sera, "A variational approach to homoclinic orbits in Hamiltonian systems," Mathematische Annalen, vol. 288, no. 1, pp. 133-160, 1990.

[5] V. C. Zelati and P. H. Rabinowitz, "Homoclinic orbits for second order Hamiltonian systems possessing superquadratic potentials," Journal of the American Mathematical Society, vol. 4, no. 4, pp. 693-727, 1991.

[6] E. Séré, "Existence of infinitely many homoclinic orbits in Hamiltonian systems," Mathematische Zeitschrift, vol. 209, no. 1, pp. 27-42, 1992.

[7] P. Caldiroli and P. Montecchiari, "Homoclinic orbits for second order Hamiltonian systems with potential changing sign," Communications on Applied Nonlinear Analysis, vol. 1, no. 2, pp. 97-129, 1994.

[8] Y. Ding and L. Jeanjean, "Homoclinic orbits for a nonperiodic Hamiltonian system," Journal of Differential Equations, vol. 237, no. 2, pp. 473-490, 2007.

[9] Y. Ding and C. Lee, "Existence and exponential decay of homoclinics in a nonperiodic superquadratic Hamiltonian system," Journal of Differential Equations, vol. 246, no. 7, pp. 2829-2848, 2009.

[10] G. Fei, "The existence of homoclinic orbits for Hamiltonian systems with the potentials changing sign," Chinese Annals of Mathematics. Series B, vol. 17, no. 4, pp. 403-410, 1996.

[11] M. Izydorek and J. Janczewska, "Homoclinic solutions for a class of the second order Hamiltonian systems," Journal of Differential Equations, vol. 219, no. 2, pp. 375-389, 2005.

[12] Y. Lv and C.-L. Tang, "Existence of even homoclinic orbits for second-order Hamiltonian systems," Nonlinear Analysis. Theory, Methods \& Applications, vol. 67, no. 7, pp. 2189-2198, 2007. 
[13] W. Omana and M. Willem, "Homoclinic orbits for a class of Hamiltonian systems," Differential and Integral Equations, vol. 5, no. 5, pp. 1115-1120, 1992.

[14] P. H. Rabinowitz, "Homoclinic orbits for a class of Hamiltonian systems," Proceedings of the Royal Society of Edinburgh. Section A, vol. 114, no. 1-2, pp. 33-38, 1990.

[15] P. H. Rabinowitz and K. Tanaka, "Some results on connecting orbits for a class of Hamiltonian systems," Mathematische Zeitschrift, vol. 206, no. 3, pp. 473-499, 1991.

[16] A. Szulkin and W. Zou, "Homoclinic orbits for asymptotically linear Hamiltonian systems," Journal of Functional Analysis, vol. 187, no. 1, pp. 25-41, 2001.

[17] K. Tanaka, "Homoclinic orbits in a first order superquadratic Hamiltonian system: convergence of subharmonic orbits," Journal of Differential Equations, vol. 94, no. 2, pp. 315-339, 1991.

[18] X. H. Tang and X. Lin, "Homoclinic solutions for a class of second-order Hamiltonian systems," Journal of Mathematical Analysis and Applications, vol. 354, no. 2, pp. 539-549, 2009.

[19] X. H. Tang and X. Lin, "Existence of infinitely many homoclinic orbits in Hamiltonian systems," Proceedings of the Royal Society of Edinburgh. Section A, vol. 141, no. 5, pp. 1103-1119, 2011.

[20] X. H. Tang and X. Lin, "Infinitely many homoclinic orbits for Hamiltonian systems with indefinite sign subquadratic potentials," Nonlinear Analysis. Theory, Methods \& Applications, vol. 74, no. 17, pp. 6314-6325, 2011.

[21] X.-H. Tang and M. Zhang, "Lyapunov inequalities and stability for linear Hamiltonian systems," Journal of Differential Equations, vol. 252, no. 1, pp. 358-381, 2012.

[22] X. H. Tang, Q.-M. Zhang, and M. Zhang, "Lyapunov-type inequalities for the first-order nonlinear Hamiltonian systems," Computers \& Mathematics with Applications, vol. 62, no. 9, pp. 3603-3613, 2011.

[23] S. B. Eliason, "A Lyapunov inequality for a certain second order non-linear differential equation," Journal of the London Mathematical Society. Second Series, vol. 2, pp. 461-466, 1970.

[24] S. B. Eliason, "Lyapunov type inequalities for certain second order functional differential equations," SIAM Journal on Applied Mathematics, vol. 27, pp. 180-199, 1974.

[25] G. Sh. Guseinov and B. Kaymakçalan, "Lyapunov inequalities for discrete linear Hamiltonian systems," Computers \& Mathematics with Applications, vol. 45, no. 6-9, pp. 1399-1416, 2003, Advances in difference equations, IV. 


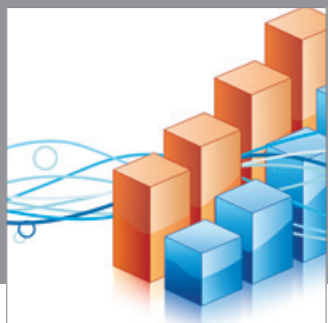

Advances in

Operations Research

mansans

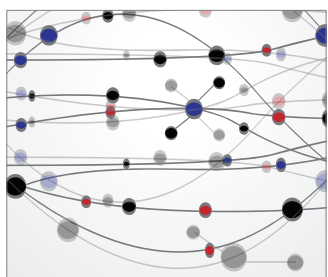

The Scientific World Journal
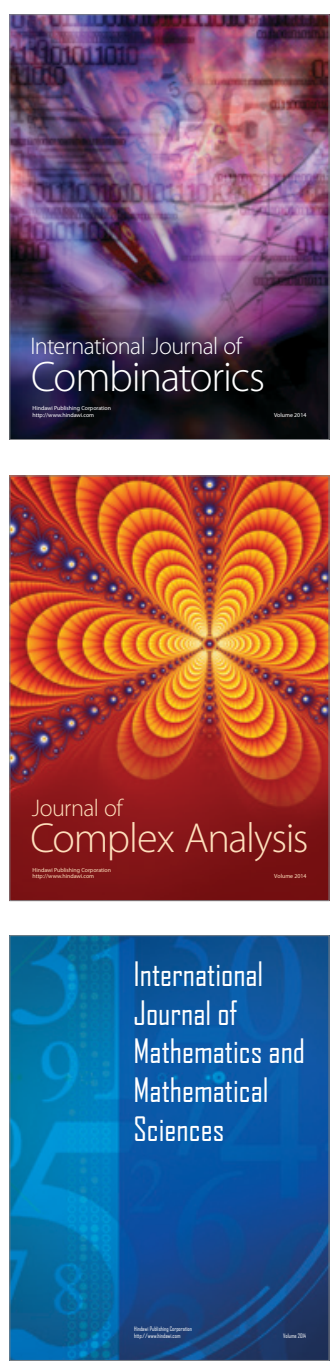
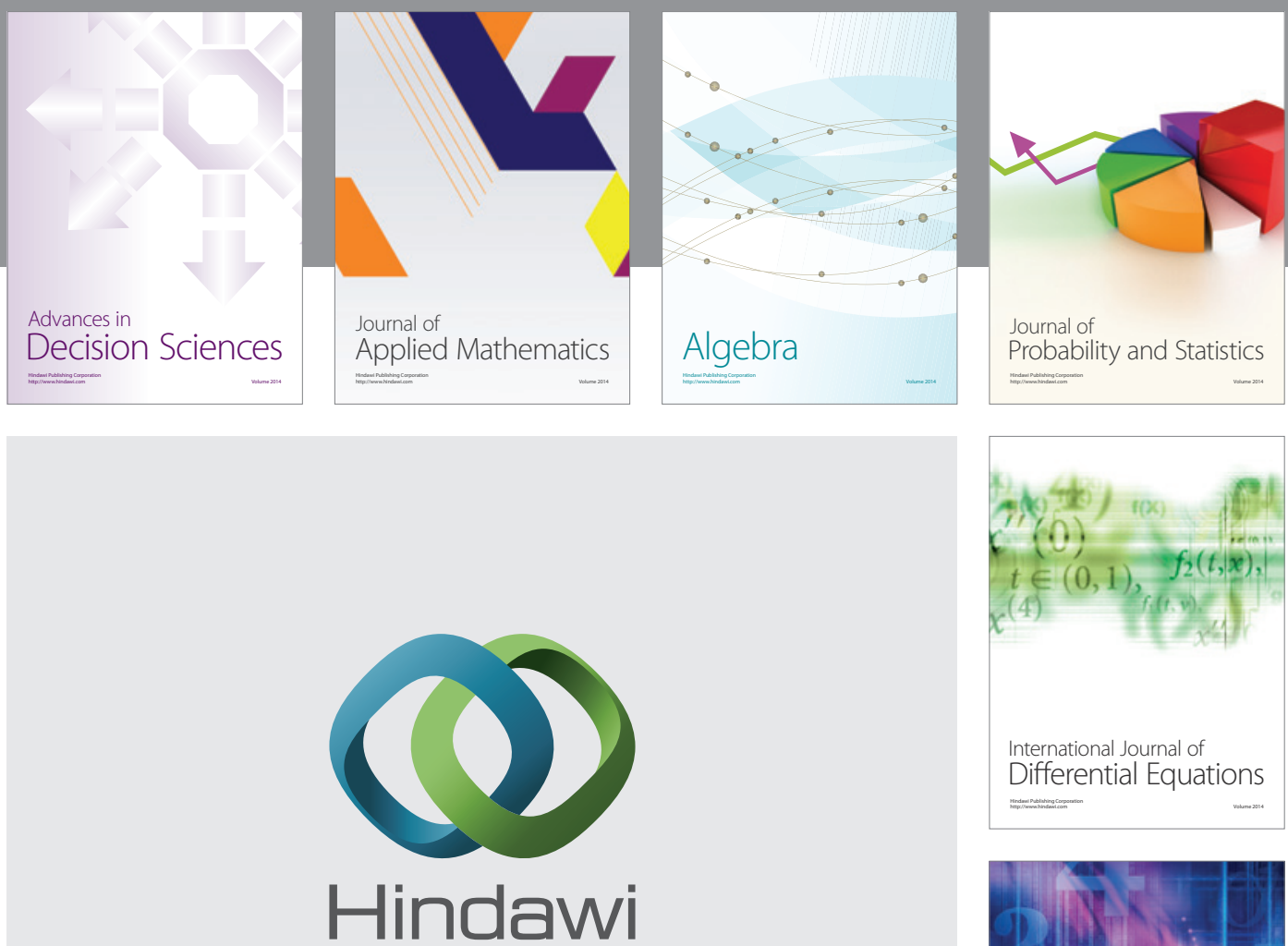

Submit your manuscripts at http://www.hindawi.com
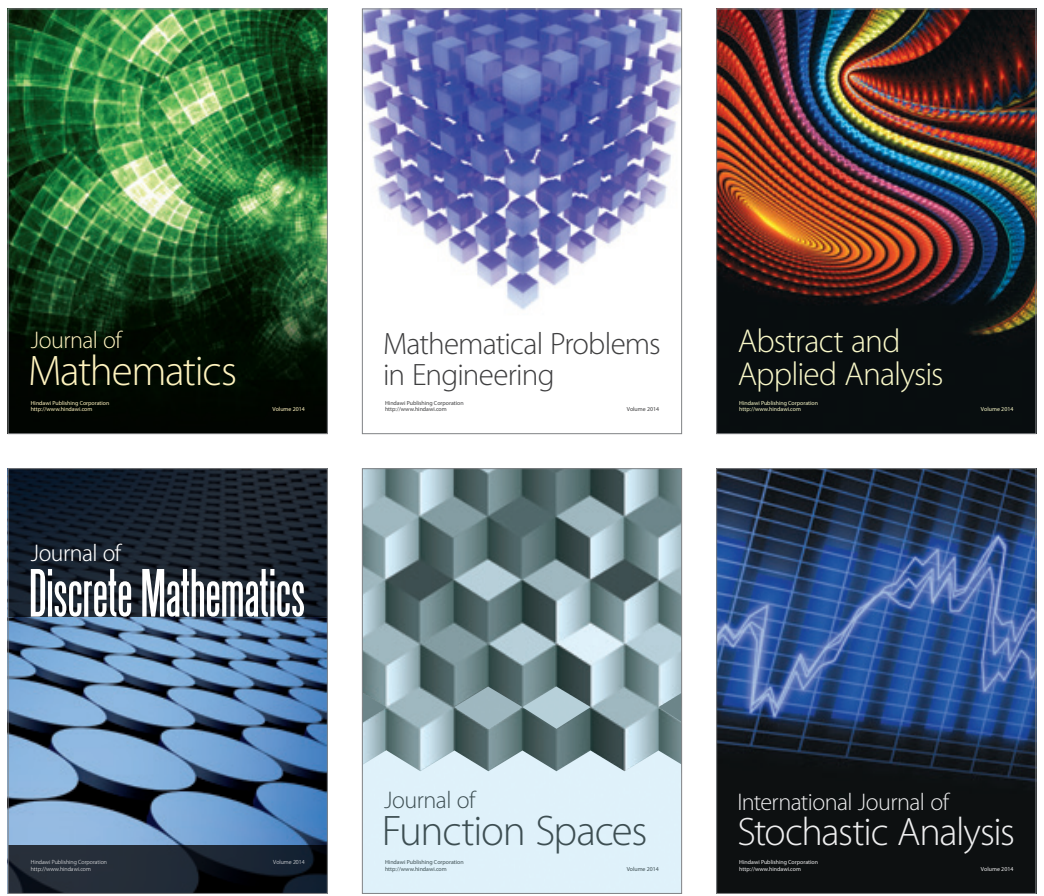

Journal of

Function Spaces

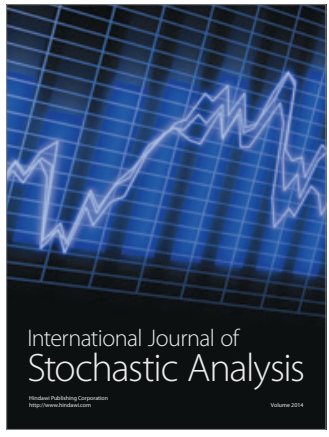

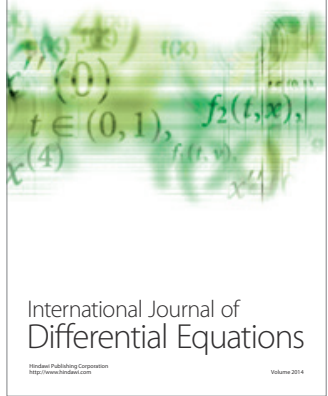
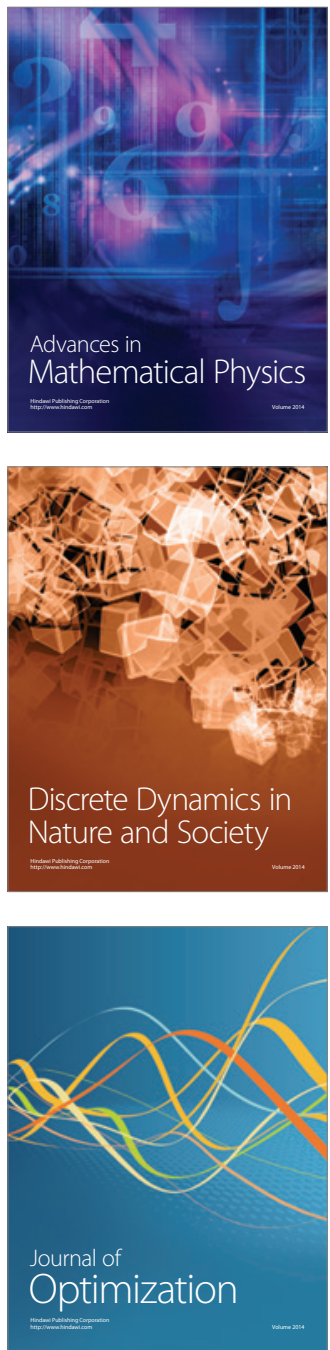